\title{
Nuevos elementos para la reflexión metodológica en sociología. Del debate cuantitativo/cualitativo al dato complejo
}

\section{Elena Jorge Sierra}

Universidad de Alicante. Departamento de Sociología I y Teoría de la Educación elena.jorge@ua.es

\section{Resumen}

El artículo aborda la problemática metodológica en sociología en torno al «eterno debate cuantitativo/cualitativo", desde una nueva perspectiva: la de la complejidad. Se trata de reavivarlo y reclamar su presencia permanente como telón de fondo de la «investigación normal» de lo social, si sirve el símil kubniano. Todo ello debe proporcionar nuevos elementos de reflexión a este debate y servir de primera aproximación que, a la luz de concepciones alternativas, nos acerque a una metodología más flexible y realista. Así, y al hilo de las ideas derivadas de la perspectiva de la complejidad y junto a principios científicos como los de indeterminación efectiva, indecibilidad, imprecisión..., se presenta una orientación metodológica que pretende superar, más que integrar, la dualidad de enfoques metodológicos en sociología. Una orientación tal, concebida a su vez como sistema complejo adaptativo, se estructura en torno al dato complejo, como elemento central del flujo de información de éste y mediador entre teoría y empiria.

Palabras clave: metodología social, cuantitativo/cualitativo, paradigma de la complejidad, sistemas complejos adaptativos, dato complejo, indeterminación efectiva, caos, indecibilidad.

Abstract. New elements for a methodological think on Sociology. From the debate quantitativelqualitative approach to the complex data

This article focuses on the sociological quantitative/qualitative dilemma form a new perspective: the complexity. The goal of this article es to provide new elements for a more flexible and creative methodology. Following this paradigm of complexity (along with other such as effective uncertainty, incompleteness, chaos, etc...), it is showed a methodological orientation in order to surpass the methodological duality approach in Sociology. This methodology is understood as a Adaptative Complex System, having the complex data as the central element of it, and as mediator between theory and empireia.

Key words: social methodology, quantitative/qualitative, complexity paradigm, adaptative complex system, complex data, effective uncertainty, chaos, incompleteness. 


\section{Sumario}

\section{Introducción}

2. De lo cuantitativo a lo cualitativo y viceversa: una dicotomía no superada

3. La perspectiva de la complejidad como metáfora para el análisis social.

La superación del debate cuantitativo/cualitativo
4. De una metodología como sistema complejo adaptativo (SCA) al dato complejo

5. Contexto metodológico y articulación del dato complejo: técnicas no paramétricas, análisis exploratorio, metaanálisis y simulaciones informáticas

Bibliografía

\section{Introducción}

La reflexión teórica sobre el método es siempre una cuestión crucial y esencial en cualquier disciplina, máxime cuando su objeto de estudio presenta unas características tan ricas y peculiares. A pesar de que para algunos se trate de una cuestión recurrente e incluso retórica, la raíz de la preocupación metodológica se encuentra íntimamente ligada al origen y desarrollo de la sociología. Así, desde su comienzo ésta surge en comunión a la idea de monismo metodológico, concepción que, derivada de la filosofía positiva, no es un hecho accidental sino consecuencia de toda una concepción anterior y un contexto sociohistórico concreto. Por contra, y al mismo tiempo, el pensamiento idiográfico reclama la singularidad y especificidad de los fenómenos no naturales (ciencias del espíritu) y se opone a la unidad de método. Como consecuencia, la aplicación del método científico y su desarrollo en sociología toma la forma de una concepción metodológica y epistemológica dual o dividida. De este modo, y mientras la tradición inglesa desde Bacon (1620) favoreció el desarrollo de orientaciones más cuantitativistas, la herencia de la filosofía idealista y el historicismo alemán fomenta un planteamiento fenomenológico y antiempirista. Así, desde la aritmética politica de Willam Petty (1676), la estadistica moral de Quetelet (1835) y los primeros análisis empíricos y estadísticos de Booth (1910), Le Play (1855) y Durkheim (1895), por una parte y la tradición historicista alemana, por otra, van sentando las bases de la actual dicotomía cuantitativo/cualitativo.

Sea como fuere, es en este momento inicial de la sociología cuando se abre una clara preocupación metodológica, que encierra en sí misma la semilla de un problema recurrente en las ciencias sociales: método y conocimiento de la realidad social. El análisis debe, pues, centrarse en averiguar que subyace en esta persistencia del problema metodológico, sus repercusiones y su conexión con el estatuto científico de la sociología y su desarrollo como tal.

La aplicación del método científico al análisis social, desde la perspectiva cuantitativa, supone la asunción de ciertos modelos matemáticos y un lenguaje axiomático generalizados como elementos de comunicación y validez cientí- 
fica. Ello confiere legitimidad y estatuto científico a la sociología, lo que a su vez valida toda su práctica científica. Así, si en origen, la preocupación por el método está íntimamente ligada a la necesidad de justificación de un determinado contexto social, del mismo modo metodología y teoría se encuentran íntimamente unidas. Por ello, el problema que aquí se plantea es la insuficiencia o no del tratamiento matemático en el análisis social para abarcar toda su complejidad y riqueza. Los fenómenos sociales, a diferencia de los naturales, hacen referencia y son fruto de procesos de autoconciencia y reflexión, en los que participa tanto el investigador como el investigado, formando parte de un todo vivo que se recrea constantemente, fruto de estos procesos de interacción consciente. Todos estos aspectos son susceptibles de tratamiento matemático, la cuestión es si, a partir de él, puede recogerse toda la multidimensionalidad y complejidad de los fenómenos sociales, debido a su particular idiosincrasia, no comparable a la de ningún otro objeto de estudio. La riqueza, complejidad y la heterogeneidad epistemológica que lo caracterizan, no puede abarcarse ni se agota con la simple emulación del modelo metodológico de las ciencias naturales. De ello se derivan las dificultades de aplicación de dichos modelos matemáticos, al ser el mundo social un sistema abierto y no un todo cerrado de verdades lógicas y axiomáticas. Muy por el contrario, puede pensarse que esta complejidad es sólo aparente y que la naturaleza de la misma, no es cualitativamente diferente a la del cualquier otro tipo de fenómeno, por lo que puede abarcarse a partir de los modelos matemáticos y axiomas adecuados. De este modo y desde una visión holística, cualquier tipo de fenómeno forma parte de un todo que responde a las mismas y sencillas reglas latentes. El hecho de que teorizaciones caracterizadas por este talante de unificación científica surjan recurrentemente, cada cierto tiempo, pone de manifiesto la transcendencia de toda esta problemática. Desde este punto de vista, esta exposición supone una crítica interna desde la perspectiva cuantitativa en cuanto al modo de investigar a partir de este enfoque. Se trata de analizar el estilo ritualista e instrumental derivado parcialmente de la aplicación de los modelos matemáticoestadísticos al análisis social, y los supuestos teóricos bajo los que se realiza su aplicación. El problema no es tanto si la acción social puede o no medirse, cuantificarse o analizarse, de acuerdo o no, con los modelos matemáticos existentes, sino bajo qué supuestos se realiza su aplicación y las repercusiones, metodológicas y teóricas, que de ello se derivan. Respecto a ello, las cuestiones referentes a la medición y cumplimiento de los supuestos paramétricos ponen de relieve toda la problemática de la hipótesis central del isomorfismo para su aplicación. La temática en torno a la utilización de determinadas técnicas de análisis, no diseñadas inicialmente para el estudio de fenómenos sociales, no está en los instrumentos es sí, sino en los supuestos que guían y aconsejan su uso y su modo de utilización. Con ello queremos decir que el problema no es la utilización de ciertos modelos matemáticos, sino su adecuación. En este sentido, se antepone su utilización por la legitimación interna y externa que confieren a la ciencia social: internamente como prueba de validez para conseguir su estatuto científico, y externamente con el resto de la sociedad para legitimar 
ciertos resultados que conllevan actuaciones concretas. Es decir, parece que el fin en sí mismo es la utilización de dichos modelos independientemente de su adaptación real al estudio de los fenómenos sociales. Fijémonos, por ejemplo, en los análisis estadísticos más frecuentes, regresión múltiple, Anova, análisis factorial, todos ellos derivan de la asunción del modelo lineal general, lo que supone asumir sin discusión que los procesos sociales pueden explicarse a través de modelos lineales, en los que ha de respetarse, entre otros, el supuesto de incorrelación - no multicolinealidad- entre las variables y/o factores. La asunción de este modelo lineal supone que la explicación de los fenómenos sociales puede hacerse a partir de diversas variables cuyo efecto aditivo ofrece como resultado el fenómeno en cuestión. Todo ello conlleva, además, la suposición de que el proceso o fenómeno social que estudiamos es un todo caracterizado por la suma de las partes. Esto deja de lado la realidad de que las variables que explican cualquier fenómeno están ineludiblemente relacionadas entre sí y el resultado de su combinación es diferente a la suma de las partes, o efecto de cada una de ellas - lo que denominamos sinergia social- Si la realidad social no encaja en los modelos y análisis estadísticos al uso, ésta se «recorta» para hacerla «encajar» en ellos. De este modo, sólo podemos obtener lo que pretendemos, la idea y la explicación de los procesos sociales en términos lineales. Una realidad que no aparezca como regular, causal, determinista y no concuerde con los modelos asumidos, simplemente "no existe». En este sentido y con R. Collins (1984), pensamos que «el gran error es ver la estadística simplemente como un método neutral, ya que, como tal, está contaminada de presupuestos teóricos ocultos» ${ }^{1}$. De este modo, nuestra aproximación y conocimiento de la realidad social se delimita y se circunscribe a unos parámetros que no pueden recoger su complejidad, dando lugar a explicaciones redundantes pero «vacías».

\section{De lo cuantitativo a lo cualitativo y viceversa: una dicotomía no superada}

Argumentaciones de este tipo y alternativas encontradas están en el substrato de la polémica cuantitativo/cualitativo, como manifestación de esta problemática. Pero la raíz del enfrentamiento de estas perspectivas no es meramente instrumental ni técnica como generalmente se presenta, sino esencialmente epistemológica. Concepciones divergentes sobre la naturaleza de lo social dan lugar a dos aproximaciones metodológicas que, bajo sus respectivos supuestos reduc-

1. La etimología de la misma expresión "estadística» ya debiera prevenirnos; «estadística» deriva de «Estado». Si bien es cierto que no debe de confundirse la parte con el todo, que la estadística es una disciplina relevante, no deben olvidarse nunca ciertas tradiciones socioculturales y claves en los números que se utilizan como «materia» de investigación. Una cita reveladora a este respecto, es la que Leslie Kish (1995: 182) incluye en su libro Diseño estadístico para la investigación: "Los gobiernos ponen mucho entusiasmo en acumular estadísticas [...]. Pero nunca debes olvidar que cada una de estas cifras proviene, en primera instancia, del alguacil del pueblo, que apunta lo que le viene en gana» (Sir Joseph Stamp). 
cionistas, se preocupan más por autoafirmarse que por resolver el problema metodológico de fondo. Planteado en estos términos, el debate cuantitativolcualitativo se centra en la cuestión de validez de ambos enfoques en pugna por la legitimación de idoneidad de sus planteamientos para el análisis social. La consecuencia es que esta preocupación, tanto en una orientación como en otra, se hace tan central que relega las cuestiones sustantivas de la reflexión metodológica. Sentadas así las bases de la polémica, ésta parece irresoluble, aunque no pocos han sido los autores que, desde distintos enfoques, reclaman una solución que pasa por la complementariedad evidente ${ }^{2}$ de ambas perspectivas. Las aportaciones en este sentido son tan diversas como distintos los puntos de vista desde los que se plantean, pero todas tienen en común una misma base argumental; la insuficiencia de la exclusividad metodológica de una u otra perspectiva para el análisis de la realidad social caracterizada por su diversidad y complejidad. A pesar de este convencimiento común, los resultados de estos planteamientos se han materializado más en una solución de compromiso que en una superación real del problema de fondo. Ello es debido a que la solución se pretende aplicar sobre las manifestaciones y consecuencias del problema, en lugar de abordar su origen epistemológico. Así, todos estos intentos de integración no han dado como resultado la superación de la dicotomía metodológica, ni han supuesto la adopción de una nueva síntesis; muy por el contrario, se han visto reducidos a sus aspectos más superficiales y meramente instrumentales. El motivo de todo ello se encuentra en el nivel al que se plantea o se entiende esta complementariedad, pues ésta se concreta, la mayor parte de las veces, respecto a cuestiones técnicas y de procedimiento. Esto pone de manifiesto que la concepción del enfrentamiento entre ambas perspectivas, cuantitativa/cualitativa, se halla en el substrato tecnológico de la investigación. $\mathrm{Y}$ es precisamente aquí donde se encuentra la quiebra de este planteamiento

2. Actualmente, encontramos un compendio de posturas respecto a la estrategia de integración metodológica en la investigación social, que van desde la negativa más absoluta - cuantiattivistas y cualitativistas recalcitrantes - hasta los mas partidarios del multiparadigmatismo metodológico, pasando por las estrategias generales de complementación, combinación y triangulación, la complementariedad por deficiencia que propone Alfonso Ortí (1994) y la "tercera vía», de los diseños multimétodo (Bericat, 1998: 56). Como el mismo autor señala, las bases de esta nueva perspectiva de integración deben ser «la prudencia metodológica» y «el principio de utilidad». En sus palabras: «Sin duda, la distinción entre las orientaciones metodológicas cuantitativa y cualitativa constituye un buen ejemplo del código binario, excluyente, que no logra reducir la realidad a sus categorías sino a costa de una insatisfactoria simplificación. La divisoria entre los métodos cualitativo y cuantitativo es la expresión bifurcada de la complejidad social».

Bericat representa la disyuntiva cuantitativo/cualitativo a través de una metáfora que favorece su compresión y análisis. Esta dicotomía se configuraría a partir de dos pirámides enfrentadas, que él denomina la doble pirámide de la investigación social. Esta dualidad metodológica, con claros antecedentes en los albores de la sociología, hace necesaria una deconstrucción de la misma en aras a su integración. Así, y dentro de este contexto argumental, los diseños multimétodo se configuran como la estrategia de integración más adecuada. 
de integración y se manifiesta su incapacidad para resolver el problema. Al contrario de lo que se presume, la diferencia esencial entre estas dos orientaciones metodológicas es la existencia de una discontinuidad teórica que afecta al contenido mismo de la investigación, y no sólo a simples problemas de procedimiento.

Pero en esta polémica no sólo está en juego la cuestión de adecuación e idoneidad metodológica de uno u otro enfoque, sino también la competencia por el estatuto de cientificidad de una u otra concepción. Así, los argumentos en favor de la síntesis y complementariedad de perspectivas no son percibidos de igual modo por los partidarios de una y otra perspectiva. Desde un enfoque cuantitativo, la lectura que se hace de cualquier intento de complementariedad es la de una concesión de legitimidad cientifica, ya que sólo se considera la validez y significación ${ }^{3}$ científica y analítica, en función de criterios estadísticos.

Por todo ello, nuestra argumentación en torno a esta problemática pone el tono en la raíz epistemológica del conflicto cuantitativo/cualitativo, y señala como horizonte adecuado para su superación un planteamiento radical, a partir del cual este debate pierda sentido y no quede relegado a una mera suma de ambas orientaciones. Se suele considerar que la complejidad del objeto se corresponde con un pluralismo epistemológico y por ende con un pluralismo metodológico. La alternativa que proponemos es abordar esta complejidad desde una epistemología, la de la complejidad, y de ahí desarrollar una única orientación metodológica con el dato complejo como elemento articular de la relación entre teoría y empiria. Este planteamiento supone un nuevo punto de partida que sustituye dos concepciones estrechas y enfrentadas de la realidad social por una más abierta aunque comprometida y difícil de abordar metodológicamente. Ello conlleva una concepción de la realidad social, como una realidad compleja y multidimensional; la epistemología de lo social, es una epistomología de la complejidad. Es decir, la esencia y naturaleza de lo social es su complejidad, y no tanto sus aspectos y manifestaciones concretas y mensurables, de un lado, y la significación latente de la acción social, de otro. Como decimos, un planteamiento tal elimina la posibilidad de una epistemología dividida y una dualidad metodológica que pretende resolverse al fin con una síntesis instrumental de ambas perspectivas. Desde este punto de vista, la complejidad de lo social, fruto de la interaccción de sus múltiples dimensiones, debe abordarse desde una metodología amplia y abierta, capaz de abarcar de manera unificada la multidimensionalidad de los fenómenos sociales. Con este fin, ha de desarrollarse un elemento de integración que concrete, articule y haga posible el análisis y la comprensión de los fenómenos sociales; concep-

3. Se encuentran incluso algunos enfoques pragmáticos como el de Gary King, Robert O. Keohane y Sidney Verba (2000: 13), que pretenden una lógica inferencial única para ambas perspectivas metodológicas. Para estos autores las diferencias entre ambas perspectivas son sólo de estilo e instrumentales, ya que en ambas subyace una misma lógica que genera inferencias igualmente válidas. 
ción que nosotros hemos denominado dato complejo. El dato complejo es un elemento integrador de los aspectos cuantitativos y cualitativos de lo social, que contempla tanto los aspectos mensurables como los no directamente observables, los más latentes, abstractos e indeterminados. Para llevar a cabo un desarrollo semejante, es necesario un contexto teórico y metodológico que asuma la influencia de los elementos caóticos, de incertidumbre e indeterminación ${ }^{4}$, en la formación y transcurrir de los fenómenos y procesos sociales, aspectos integrantes, a su vez, de la caracterización del dato complejo.

\section{La perspectiva de la complejidad como metáfora para el análisis social. La superación del debate cuantitativo/cualitativo}

Con un convencimiento tal, y al hilo de los actuales desarrollos de la teoría de la complejidad ${ }^{5}$, adoptamos este marco de referencia como metáfora respecto a lo social, lo que nos ayudará a replantear la raíz del debate cuantitativo/cualitativo en un nuevo punto de partida: el de la complejidad. Lejos de dejarnos arrastrar por lo que muchos han denominado el silogismo seductor, proponemos esta metáfora de la complejidad como elemento dinamizador de nuestra metodología con objeto de ampliar el marco de reflexión del debate cuantitativo/cualitativo, y abrir la puerta a una visión y un tratamiento metodológico más rico y creativo, a la vez que realista. Y consideramos que no es adecuado adoptar

4. Estos principios de indeterminación cuántica e indeterminación efectiva, presentes conceptualmente en la teoría del caos, se refieren a que los procesos caóticos presentes tanto en la naturaleza como en el mundo social, funcionan de tal modo que un mínimo cambio en las condiciones iniciales de cualquier proceso puede producir un fenómeno radicalmente diferente al esperado.

5. En los últimos años vienen desarrollándose en torno al Instituto de Santa Fe, encabezadas por Murray Gell-Man (1995), las más interesantes reflexiones y avances en relación con los estudios de la complejidad. A pesar de ello, este centro no monopoliza el estudio sobre esta temática; recordemos las aportaciones de Ilya Prigogine (1994) y otros. Esta orientación con vocación de unificación científica supone una nueva aproximación al conocimiento basado en la idea de complejidad. La intención última que guía estos estudios es la de construir una teoría unificada de los sistemas complejos adaptativos. Esta construcción parte de la hipótesis de que es posible el descubrimiento de unos principios generales que rigen el funcionamiento de los mismos. Desde este punto de vista, los sistemas complejos adaptativos (SCA) se conciben como sistemas fruto de la aleatoriedad y la regularidad, que manejan flujos de información ofreciendo respuestas cada vez más adaptadas y exitosas en relación con su medio y su propio funcionamiento. Estas respuestas son consecuencia de las esquematizaciones obtenidas de regularidades existentes en los flujos de información, pero son esquemas que deben completarse con los elementos no regulares, accidentales o aleatorios de dicha información. De este modo, dichos esquemas sujetos a variación son puestos a prueba en su contraste con la realidad. Así, en este proceso, los esquemas se contrastan con datos de la misma naturaleza del esquema extraído, de modo que lo que ocurre en el mundo real determina qué esquemas sobreviven. Estos sistemas, pues, requieren para su existencia condiciones medias entre el orden y el desorden. El estudio del procesamiento de los flujos de información debe permitir extraer las «leyes» que rigen el funcionamiento de los citados SCA. En definitiva, la premisa central de esta perspectiva es que la configuración y el mantenimiento de los fenómenos complejos está regulado por reglas simples. 
la perspectiva de la complejidad en un sentido global, porque ello supondría asumir de nuevo los presupuestos de los modelos matemáticos que lleva aparejados sin que se hayan resuelto en nuestra disciplina los problemas teóricos y de isomorfismo en torno a esta cuestión.

Como bien apunta Edgar Morin (1995: 87-89), «la idea de complejidad no es una idea moderna fruto de los nuevos desarrollos científicos, pues si bien es cierto que desde el siglo XIX la ciencia, bajo un paradigma de la simplicidad, pretendía el descubrimiento de un mundo determinista basado en la causalidad de leyes generales, la literatura mostraba lo singular, identidades individuales en un tiempo y un espacio concretos».

Así, cuando decimos que utilizamos la teoría de la complejidad como metáfora para el análisis social, nos estamos refiriendo a que adoptar ésta en un sentido estricto, nos lleva parcialmente al determinismo que queremos evitar. Asumir esta convicción en su expresión matemática supone admitir que ecuaciones deterministas están en la base de todo fenómeno complejo, sea cual sea su naturaleza. Cuando Edward Lorenz, en 1967, estudiaba el comportamiento de la atmósfera simple, descubrió que éste, en apariencia desordenado, irrepetible e impredecible, podía explicarse mediante un sistema de tres ecuaciones completamente determinista. Así, la lógica del concepto de caos determinista va intrínsecamente unida a la latencia de leyes simples que explican el funcionamiento de determinados procesos.

La descripción anterior parece adecuada a la expresión y apariencia de los procesos sociales, por lo que la idea de tratarlos de un modo semejante resulta muy atractiva y pertinente. Pero como ya dijimos antes, el desarrollo teórico en sociología no permite establecer una continuidad de método entre lo físico y lo social, por lo que dejarnos llevar por este silogismo seductor no resuelve nuestros problemas a ningún nivel. Además, esta pretensión sintetizadora en términos algorítmicos, que parece en última instancia latente a diversos planteamientos de unificación científica, que aparecen de modo recurrente cada cierto tiempo en la ciencia, no es del agrado de todos. En contra de la idea de una matematización omnipresente, se forja una de las críticas más coherentes dirigida a los estudios de la complejidad. Ésta se centra en la idea de que la asunción de los principios anteriores supone una matematización última de todos los fenómenos, que sólo es aplicable y verificable en sistemas «cerrados» con una lógica matemática intrínseca. Para el análisis del resto de los sistemas "abiertos», como es el sistema social, la aplicación de modelos matemáticos pierde vigor o se hace más complicada y problemática. Por ello, la adopción de la perspectiva de la complejidad es más una metáfora que una aplicación directa con implicaciones matemáticas concretas.

Una solución a este dilema sería la adopción de una nueva perspectiva, que pudiera combinar la asunción de nuestros límites al conocimiento sin perder de vista el objetivo y el deseo de acercarnos cada vez más a él. En este sentido, algunos autores sugieren que la ciencia puede hacerse menos lineal y más poética. La poesía constituye un uso muy poco lineal del lenguaje; en ella, el significado es más que la suma de las partes. Aplicar esta idea a nuestra disciplina 
podría llevarnos a la adopción de un modelo de análisis y explicación de lo social en términos narrativos; que permita reflejar adecuadamente la esencia y naturaleza de lo social.

Además, y con Anderson (1972), pensamos que la vida está modelada en menor medida por leyes deterministas que por contingencias y circunstancias impredecibles. En este sentido, lo importante a destacar es que asumir de uno u otro modo el modelo de la complejidad y los conceptos e ideas con ella relacionados puede ser una aventura interesante que ayude a la superación de ciertas concepciones y prácticas que dificultan y frenan el avance de nuestra disciplina, pues condicionan negativamente nuestra visión metodológica, como se refleja en el enfrentamiento cuantitativo/cualitativo. Estas consideraciones, junto a la aplicación de una perspectiva de la complejidad al mundo de $l o$ social, fortalecen la idea de desarrollar y articular la noción de dato complejo.

Sea como fuere, y sin hacer oídos sordos a estas críticas, los estudios sobre la complejidad se dibujan, a nuestro juicio, como una alternativa prometedora para el estudio de lo social, y, desde este punto de vista, vamos a considerarlo en nuestro modelo de lo que hemos dado en llamar dato complejo. Desde esta perspectiva, la realidad social sería uno de los múltiples sistemas complejos adaptativos situados entre la regularidad y el caos. La asunción de una perspectiva semejante, sin duda, modificaría en gran medida muchas de nuestras concepciones y modos de investigar lo social. Y esto es precisamente lo que aquí queremos destacar; puede que la perspectiva de la complejidad resulte ser una entelequia con conceptos y nociones vacías de contenido, pero la posible aplicación de esta orientación a nuestra disciplina pretende ser, para nosotros, más "pedagógica» que esencialmente transformadora. Con esto queremos decir que el hecho de considerar orientaciones de este tipo, sin duda, puede ayudar a la superación de modos de pensamiento y prácticas metodológicas decimonónicas, cargadas de supuestos dualistas, deterministas, mecanicistas y de visiones parciales. En este sentido, nos situaríamos en la línea de las ideas de Anderson (1972) recogidas en su ensayo More is diferent, sobre la limitación de los enfoques reduccionistas para explicar el mundo. Aunque, al mismo tiempo, señala que esta concepción no tiene necesariamente que desembocar en una teorización sobre los sistemas complejos. "No creo que haya una teoría del todo [...] Sí creo que existen principios básicos de muy amplia generalidad, como la mecánica cuántica, la mecánica estadística, la termodinámica y la ruptura de la simetría. Pero no se debe caer en la tentación de creer que, cuando se tiene un buen principio general en un nivel, va a funcionar en todos los niveles.»

Aun dejando la puerta abierta a toda hipótesis de este tipo, nosotros abordamos la complejidad para el estudio de lo social en el sentido de que consideramos la estructura y naturaleza de lo social como fruto de procesos de orden y de desorden en el límite del caos. Desde este punto de vista, adoptar la perspectiva de la complejidad supone asumir la contradicción, lo diverso, lo que parece consecuencia del azar o el desorden, al mismo tiempo que la síntesis que no diluye en ella a los contrarios. El descubrimiento de contradicciones 
es inherente a cualquier fenómeno complejo, por lo que su detección no es señal de error, sino que se valora su capacidad heurística para enriquecer la compresión y el conocimiento, en definitiva, el reconocimiento de la contradicción incita a un afán de superación de la misma pero que no la niega. La metáfora de la complejidad, además, supone aceptar nuestros límites al conocimiento bajo los supuestos de imprecisión, indecibilidad e incompletud ${ }^{6}$. Nos sitúa, así, la metáfora de la complejidad en una posición incómoda respecto a los hábitos y prácticas de pensamiento científico tradicional, ya que niega la posibilidad de certidumbre y de totalidad pero sin refugiarse en un relativismo absurdo. Esto implica soportar una tensión que empuja al límite del conocimiento sin reconocer la posibilidad de llegar a él en términos de certeza total.

$\mathrm{Al}$ igual que los intentos anteriores como teoría de sistemas, cibernética, teoría de catástrofes, caos, fractales, etc., la teoría de la complejidad tiene una vocación científica global y totalizante de unificación. Pero la peculiaridad de la naturaleza de lo social, a diferencia de los fenómenos que sirven de objeto a otras ciencias, hace más idónea su aplicación en sociología como metáfora didáctica que como planteamiento esencialmente transformador en toda su extensión. Así, y del mismo modo que el excesivo fervor por los nuevos modelos en otros campos científicos ha dado lugar a actitudes miméticas en sociología que no han resultado tan fructíferas como se esperaba, el alejamiento y la cerrazón a los mismos puede resultar igualmente desafortunado e inconveniente, anclando su desarrollo teórico y metodológico. Por ello, y desde una postura más moderada, conscientes de estas contradicciones y limitaciones pero sin desoir las nuevas tendencias en el ámbito general de la ciencia, planteamos la metáfora de la complejidad en el análisis social. En un momento en que, incluso desde los «santuarios» de la ciencia clásica se reclaman actitudes creativas y de estilo narrativo en la práctica científica, el maniqueismo y la dicotomía metodológica de la sociología aparece trasnochado y estéril. Del mismo modo, el intento denodado por la emulación del más puro estilo «cientifista» de las "ciencias duras», desde posturas cuantitativas, que se concreta en una obsesión por la precisión en la medida, el control y la aplicación de modelos matemáticos cada vez más sofisticados, sin conocer realmente su finalidad

6. El concepto de indeterminación de Werner Heisenberg (1958), relacionado con el contexto de la mecánica cuántica, postula la imposibilidad de conocer simultáneamente y con exactitud la posición y el momento de una partícula, lo que pone en cuestión las bases más sólidas de la física tradicional. Por otra parte, el concepto de indecibilidad de Kurt Gödel (1980), demuestra la imposibilidad de desarrollar un sistema de axiomas coherente de tal modo que de él pudiera derivarse verdad o falsedad de todas las proposiciones matemáticas consecuentes del mismo. Este principio constata el hecho de que, dado un sistema de axiomas matemáticos, no todas las proposiciones de él derivables son «decibles», es decir, siempre habrá proposiciones indecibles en relación con las que no se puede demostrar su verdad o falsedad. Esto significa que podemos obtener "verdades», pero no verdades demostrables en términos absolutos. Todo sistema intelectual (numérico, simbólico o léxico) es incompleto respecto al sistema real de referencia. Incompletitud, pues, en el sentido de que ninguna teoría puede contener todos los enunciados demostrables y verdaderos posibles. 
última —excepto su valor de legitimación científica-, suponen un ejemplo más del reduccionismo de la metodología social. Por otra parte, y desde una perspectiva cualitativa, la sistemática negativa a la aplicación del lenguaje matemático para el análisis sociológico, supone una concepción de lo social igualmente estrecha que sólo asume los aspectos cualitativos, fruto de procesos de reflexión. Desde este punto de vista, la realidad social se construye a partir de la interacción social estructurada por el lenguaje, realidad que se produce y se reproduce constantemente en un proceso de reflexión incesante, de modo que el tratamiento matemático no es el más adecuado a una naturaleza tal.

Estas posturas enfrentadas sin aparente solución de continuidad se recrudecen en el marco de una sociología en crisis permanente, y en un periodo de profundos cambios y transformaciones. En este contexto, la característica emblemática de la perspectiva cuantitativa es su hipertrofia técnica en detrimento de aspectos esencialmente metodológicos, desde una sociología de la estructura y sistema social sin sujetos. Estos meros agregados son las partes que por adición forman un todo. Por otro lado, la perspectiva cualitativa reclama los aspectos subjetivos y reflexivos de la acción desde una sociología del actor, una sociología del sujeto sin sistema.

Por el contrario, observar la realidad social como un proceso objetivo y reflexivo a la vez, entre tantos otros procesos que la conforman, supone considerarla desde un punto de vista multidimensional, en definitiva desde la complejidad. Del mismo modo, la concepción de «dato simple» (unidimensional), fruto de ambas visiones, pierde sentido y tiende hacia el horizonte de la consecución del dato complejo, que refleja esta visión multidimensional donde se unen los contrarios sin negarse.

Desde la metáfora de la complejidad, la realidad social como SCA se concibe como sistema cuyo funcionamiento es fruto de la aleatoriedad y la regularidad, del orden y del azar. Es decir, el sistema social, como otros tantos sistemas complejos adaptativos, necesita para el mantenimiento y desarrollo de sus funciones la coexistencia de orden y desorden, punto crítico que se ha denominado el filo o borde del caos. Bajo estas premisas, el conocimiento de la estructura básica de las interacciones que se desarrollan en un contexto tal, transido por elementos de regularidad y caos, es suficiente para descubrir las reglas básicas del funcionamiento del sistema, lo que nos remite a la hipótesis central de que lo que se encuentra tras la complejidad son las leyes simples de su funcionamiento.

Por ello, las esquematizaciones obtenidas a partir de las regularidades contenidas en los flujos de información del sistema son caracterizaciones insuficientes que deben completarse con los efectos aleatorios, no regulares y caóticos de dicha información. Una concepción semejante transforma en gran medida, a la vez que flexibiliza, nuestra consideración de los procesos de investigación y teorización. El descubrimiento de regularidades relativas al funcionamiento de fenómenos y procesos sociales, como base del establecimiento de «leyes» para la estructuración de un corpus teórico sólido, abre paso a una nueva concepción en la que la consideración de las irregularidades en la frontera del caos es un elemento esencial. 
En definitiva, esta concepción de la realidad social como un sistema complejo supone, además de admitir una visión sistémica, la percepción de las estructuras y los procesos sociales, no sólo caracterizados por la regularidad, sino como fruto de un funcionamiento efectivo y real, en el que intervienen elementos y procesos aleatorios y no lineales. Todo ello empuja a una transformación de nuestras concepciones y modos de investigar lo social que se dirige hacia el desarrollo de modos y maneras más abiertas, creativas y flexibles. Como ya hemos dicho, en este marco, principios científicos generales como los de indecibilidad, incertidumbre e indeterminación en relación con lo social, comprendido como realidad compleja y multidimensional, adquieren especial relieve.

\section{De una metodología como sistema complejo adaptativo (SCA) al dato complejo}

Derivada de toda esta concepción y condicionada por ella, la idea de una metodología realista en las ciencias sociales aparece unida a la caracterización y del desarrollo de la idea de dato complejo como elemento de conexión entre teoría y empiria.

Desde nuestro punto de vista, lo social se configura como hecho y como proceso, cargado de significado a la vez, asumiendo al mismo tiempo la comunión de desarrollos inductivo-deductivos para su comprensión y explicación como binomios inseparables. Elementos dinámicos como el tiempo — sincronía/diacronía- - y los diferentes contextos explicativos también han de estar presentes en esta caracterización. Al mismo tiempo, hemos de adoptar una visión global macro que no desatienda lo micro en el sentido de inclusión simultánea a modo de una concepción fractal de los procesos sociales. Todo ello en síntesis con la asunción de los principios de indeterminación, incertidumbre, indecibilidad y caos, dirigidos hacia la formulación teórica, son elementos esenciales y característicos de esta configuración del dato complejo.

Todo ello puede comprenderse mejor a través del esquema 1.

1) En primer lugar, partimos de una concepción de la realidad social, que, como sistema complejo, encierra dos aspectos fundamentales: multidimensionalidad y articulación de la superposición y relación de dichas dimensiones, que condiciona la configuración del dato complejo en esta dirección.

2) De esta realidad se obtienen datos complejos que estructuran la base del flujo de información del sistema. De un lado, la noción de dato hace referencia a una unidad básica de información dentro del proceso metodológico y, de otro, la acepción de complejo se orienta al sentido de multidimensionalidad interrelacionada.

3) En tercer lugar, la metodología social concebida a su vez como SCA orienta el tratamiento de dichos flujos de información a la extracción de regularidades dirigida a la construcción de esquematizaciones base de los procesos de teorización. Lo característico de la metodología social como sistema 


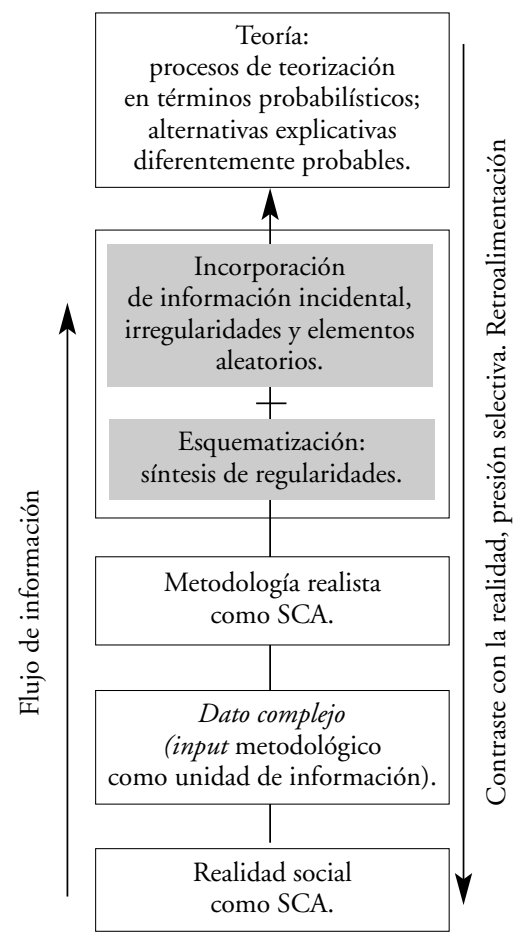

Esquema 1. El proceso de investigación científica en sociología, como sistema complejo adaptativo.

complejo adaptativo, es que todos estos procesos de teorización que posibilita no se desarrollan de manera unidireccional ni lineal, sino que, por el contrario, combinan simultánea y constantemente, la información referente a las secuencias de esquematización con las irregularidades y los elementos aleatorios. Una metodología abierta y flexible en este sentido, es capaz de recoger, a partir del dato complejo, la multidimensionalidad de los procesos sociales combinando el descubrimiento de patrones con aspectos que no reflejan regularidades.

4) Todo ello desemboca en la producción de esquemas alternativos diferentemente probables en su análisis, explicación e interpretación de lo social.

5) Estos esquemas alternativos en competencia, se someten a contraste con la realidad que ejerce una presión selectiva sobre ellos, de modo que sólo los más adecuados «sobreviven».

En nuestra argumentación, el eje central del flujo de información que retroalimenta constantemente el proceso es el dato complejo. La primera característica y cualidad de éste es su oposición al sentido reduccionista y unidimen- 
sional de "dato simple», característico de los enfoques tanto cualitativo como cuantitativo. En contrapartida con estas perspectivas, el dato complejo asume la realidad social en sus múltiples dimensiones, como hecho y como proceso, como dato y como semántica, fruto de procesos objetivos y subjetivos, de configuraciones lineales y no lineales, en fin, de procesos regulares y aleatorios que circulan en contextos espaciotemporales dinámicos.

Desde este punto de vista, el dato complejo es el elemento esencial (input) del flujo de información de la metodología social concebida a su vez como SCA. Este flujo de información, bajo el tratamiento de una metodología tal, produciría esquemas alternativos diferentemente probables, lo que nos conduce, desde este punto de vista, a una teoría probabilistica de lo social en un sentido nuevo ${ }^{7}$.

\section{Contexto metodológico y articulación del dato complejo: técnicas no paramétricas, análisis exploratorio, metaanálisis y simulaciones informáticas}

El dato complejo es un elemento pluridimensional esencial que surge no de la adición de perspectivas, sino del tratamiento simultáneo desde cada una de ellas. Es decir, podemos partir de un resultado estadístico, de un análisis documental, de uno de discurso, de un suceso accidental aparentemente inexplicable, pero indefectiblemente hemos de terminar por elaborar un dato que recoja la multidimensionalidad social y que así estructurado sirva de base al análisis. Esto nos permite trabajar con cifras a las que se imbrica su significación sustantiva real, sintetizando todo ello con el valor heurístico de los aspectos accidentales y aleatorios relacionados con los fenómenos que se analizan. En definitiva, la construcción del dato complejo es el resultado de un proceso de síntesis que no constituye el resultado final, sino la base original para el análisis. ¿Pero cuáles son las proposiciones concretas que materializan y hacen posible la consecución de datos concretos desde este punto de vista? ¿Qué instrumentos, o formas de observar y analizar la realidad social, guían la consecución de un dato con tales características, capaz de recoger la diversidad en la regularidad y todos los matices y dimensiones de lo social? ¿Qué supuestos y modo de pensar hemos de desterrar y cuáles dan forma a la base de un dato o unidad de información con tales atributos?

El problema que se presenta a partir de todo ello es cómo articular esta nueva concepción en una metodología concreta acorde con los presupuestos

7. En un interesante artículo, «Einstein, Renoir and Greeley: somo thoughts about evidence en Sociology", American Sociologycal Review, vol. 57, n. 1 , febrero de 1992, Stanley Lieberson amplía el marco teórico de la falsación popperiana, argumentando que el contraste y continuo control de los procesos de teorización, explicación y comprensión de los fenómenos sociales, debe hacerse en términos de probabilidad de ocurrencia sin desechar las cadenas de sucesos aparentemente incidentales y menos probables. Desde este punto de vista, las teorizaciones del análisis de fenómenos sociales se presentan como explicaciones diferentemente probables, sin que sucesos aislados, incongruentes o congruentes, sean hechos suficientes para su rechazo o aceptación total, todo ello sin caer en el relativismo teórico ni metodológico. 
que de ella se derivan. Se hace necesario pues, como primer paso, la revisión de las técnicas y hábitos tradicionales de investigación junto con la de los supuestos teóricos para su aplicación, al mismo tiempo que el fomento y desarrollo de todas aquellas estrategias que puedan operar de acuerdo con la noción de complejidad. Las estrategias que se presentan a continuación son algunos de los elementos que, aunque aislados, pueden constituir instrumentos destacados en conexión con el análisis desde la perspectiva del dato complejo; éstos son: el análisis exploratorio, el metaanálisis y las simulaciones informáticas.

Por todo lo anterior, la operacionalización del dato complejo como elemento articulador de una metodología realista ha de centrarse fundamentalmente en la superación de perspectivas y técnicas reduccionistas y excluyentes.

Desde un punto de vista metodológico, las vías para la obtención de un marco adecuado pasarían desde la revisión de la obra de los autores clásicos y la revisión metaanalítica de investigaciones diversas hasta la crítica concreta de investigaciones puntuales, ofreciendo una alternativa desde la complejidad. Todo ello debe acompañarse de una revisión técnica dirigida a la:

1. Crítica a los supuestos en la aplicación de las diferentes técnicas cuantitativas como los de:

- Dependencia / Independencia (no hay causalidad ni dependencia / independencia como dos polos de un continuo, pues la influencia de todas las variables es más que la suma de las partes, el hecho de que una de ellas no aparezca o la modificación en una de ellas, por pequeña que sea, puede dar lugar a procesos y situaciones totalmente diferentes).

- Control experimental/control estadístico.

- Linealidad / no linealidad.

- Niveles de medición.

- Aplicación del modelo lineal general.

- Identificación de modelos causales.

$\mathrm{Al}$ mismo tiempo que deben promoverse:

2. La profusión, el desarrollo y la utilización de las técnicas de análisis exploratorio.

3. La atención sobre los procesos metodológicos, en detrimento de los técnicos, atribuyendo a éstos sólo el valor real que puedan tener.

4. La interpretación de resultados en función de los supuestos de los que se parte en la aplicación de cada técnica concreta.

5. El desarrollo de concepciones metodológicas, basado en la articulación y superación de diferentes perspectivas metodológicas y técnicas para la consecución de datos complejos.

6. La aportación del «metaanálisis» como elemento funcional y generalizado.

7. El diseño de simulaciones y modelos informáticos ad hoc para el estudio de los fenómenos y procesos sociales. 
Análisis exploratorio, metaanálisis y simulaciones informáticas, como técnicas estratégicas en consonancia con la noción de complejidad, se convierten, así, en elementos centrales del contexto metodológico del dato complejo.

\subsection{El análisis exploratorio}

El análisis de datos desde una perspectiva exploratoria no suele ser considerado tan frecuentemente como sería deseable; y es precisamente una de las técnicas y aproximaciones más en consonancia con el pensamiento complejo. Debido a la falta de consideración de este tipo de técnica, se aplican modelos estadísticos de modo ritual sobre información que, en cierto modo, se desconoce. Adoptar un punto de partida exploratorio supone bucear en los datos y dejar que éstos expresen toda su riqueza, de modo que se muestren y hagan evidentes los patrones estructurales y relacionales subyacentes a la información que contienen. Surgen, así, las hipótesis de los datos y se amortigua el riesgo de imponer ciertos modelos de análisis no concordantes con la naturaleza de las observaciones. Además de ello, y en este marco, se evidencian de igual modo tanto las regularidades y los patrones estables implícitos en los datos (lo suave), como lo atípico o incidental, aquello que no se ajusta a los patrones de regularidad (lo áspero o rugoso).

Así, aspectos que desde otro enfoque, el de la estadística confirmatoria tradicional, serían despreciados o tratados como errores, pueden manifestar toda su riqueza heurística ofreciendo un conocimiento más aproximado y global de la realidad que se investiga. De este modo, el dilema que plantea Coombs $(1979)^{8}$ respecto al orden impuesto a los datos y el tratamiento y consideración del error, se afronta desde un nuevo punto de partida que aporta un estilo de investigar diferente. Este estilo detectivesco ${ }^{9}$,

8. En palabras de Coombs (1979: 466), según este dilema «El sociólogo se enfrenta con el problema de escoger entre poner sus datos en un orden sencillo o preguntarse si sus datos responden a un orden sencillo».

9. John W. Tukey (1977: 1-3) compara, así, el análisis exploratorio con el trabajo detectivesco: «El análisis exploratorio de los datos es un trabajo de detective — un trabajo de detective numérico, detective contable o detective gráfico-. Un detective que investiga un crimen necesita tanto herramientas como capacidad de penetración y comprensión. Si no dispone de contraste para las huellas dactilares suspenderá la búsqueda de huellas sobre las superficies que investiga. Si no conoce dónde el criminal puso probablemente sus dedos, no observará los lugares adecuados. Del mismo modo, el analista de los datos necesita de ambos; instrumentos y comprensión. "del mismo modo" [...] El proceso de justicia criminal diferencia claramente entre el análisis de la evidencia [...] y la evaluación adecuada de la misma [...] En el análisis de datos una distinción similar resulta útil. El análisis exploratorio de datos tendría un carácter detectivesco. Por su parte, el análisis confirmatorio tendría un carácter judicial o casi judicial [...] Sin el hallazgo de pistas, el trabajo judicial no tiene sentido. Sin el descubrimiento de las indicaciones del análisis exploratorio de datos, generalmente cuantitativo, es probable no considerar un análisis confirmatorio». Sherlock Holmes iría del «caso" a la regla y de la «regla» al caso, es decir, operaría "creativamente» y "exploratoriamente» combinando la inducción y la deducción en un nuevo modelo superador. 
parte de una lógica alternativa más abierta con la que se pretende descubrir las pautas latentes en los datos, buscando lo que éstos puedan revelar sin despreciar lo que parece erróneo o atípico. Desde este punto de vista, es deseable no perder la pista de los datos en ningún momento, es decir, se prima el análisis directo de éstos y no el derivado de las medidas o estadísticos numéricos, resumen de los mismos. Estos resúmenes numéricos se consideran únicamente como extracciones sintéticas de la información contenida en los datos y a menudo suelen obscurecer las características más relevantes de la información en lugar de ponerlas de relieve. Por ello, el análisis debe comenzar por los datos y no sobre un sustituto de éstos en forma de coeficientes estadísticos. Así, desde esta perspectiva, se han ideado y desarrollado toda una serie de conceptos e instrumentos de análisis que descansan en esta concepción. En síntesis: únicamente explorando los datos es posible describir lo que no se espera, es decir, los aspectos que generalmente quedan ocultos a partir de otros tipos de aproximación y que frecuentemente encierran una gran capacidad heurística que no puede valorarse si no se pone de manifiesto.

\subsection{El metaanálisis}

Respecto a otro de los elementos destacados en este marco metodológico del dato complejo, el metaanálisis sociológico destaca por su importancia como proceso de síntesis integrador de hallazgos empíricos, base de la teorización social. Nos hemos referido a él como alternativa y complemento, desde un punto de vista cuantitativo, a las versiones narrativas tradicionales. En este sentido, se trata de un tipo de análisis doblemente válido, pues, por una parte, pone en cuestión los supuestos tradicionales desde una perspectiva cualitativa y, por otra, se orienta hacia una síntesis y superación de los mismos. Este intento de integración metodológica y conceptual cuestiona los supuestos, las prácticas y las técnicas habituales que, desde la perspectiva cuantitativa, se aplican al análisis social. Así, y aunque aún perviven en el análisis metaanalítico, en la mayor parte de los supuestos habituales de la estadística tradicional, en la actualidad, se libra un debate intenso sobre los supuestos paramétricos y las aproximaciones tradicionales en este nivel de análisis ${ }^{10}$.

Eco (1989) lo ha visto muy bien en The sign of three con Thomas Sebeok en un concepto que él llama «abducción». Se trata sencillamente de «imaginación realista».

10. A este respecto, Glass (1981) recoge y sintetiza las cuatro críticas o limitaciones con las que generalmente ha de enfrentarse este tipo de orientación analítica:

1. Las relativas a la agregación de estudios con diferentes tratamientos técnicos y conceptuales. Desde este punto de vista, se mantiene que no se puede llegar a conclusiones lógicas a partir de la comparación y agregación de resultados que han sido obtenidos a partir de diferentes técnicas, muestras, variables y procedimientos.

2. Las relativas a la inclusión conjunta de diseños experimentales de calidad desigual. En este sentido, se cuestiona la validez de metaanálisis que aglutinan los resultados de estudios provenientes tanto de "pobres» como de «buenos» diseños experimentales. 
El fomento de tales procesos de síntesis del acervo empírico de nuestra disciplina, enriquecidos por esta crítica interna, se enlaza aquí con el desarrollo de una caracterización teórica probabilística, en el sentido anteriormente expuesto. El problema recurrente con el que una vez más nos enfrentamos al abordar un enfoque metaanalítico es el relativo a la debilidad conceptual y teórica de la sociología. Si la complejidad de nuestro objeto de estudio se suma a la debilidad teórica que condiciona el desarrollo y el progreso metodológico y conceptual, la alternativa a procesos de teorización hacia una teoría probabilística de lo social parece cuando menos coherente e idónea en tales circunstancias. En este sentido, el metaanálisis tiende a una síntesis global de conocimiento más que a una acumulación continua de información, es decir, esta construcción sintética a partir de gran diversidad de investigaciones empíricas supone algo más que la simple acumulación de resultados aislados, a menudo contradictorios. Ello, desde un marco de teorización probabilístico, nos lleva directamente a la consideración de alternativas explicativas y comprensivas diferentemente probables.

\subsection{Las simulaciones informáticas}

Otro de los elementos que hemos destacado como esenciales para un marco adecuado de operacionalización, desarrollo y consecución del dato complejo es el relativo a la elaboración y aplicación de simulaciones informáticas en el análisis social. Como consecuencia de la aplicación de la informática, tanto a los aspectos metodológicos como a los de teorización, difusión e intercambio de conocimientos sociológicos, se abren nuevas alternativas a los estilos y modos tradicionales de investigar. Pero, como ocurre respecto a otras técnicas, la simple aplicación de simulaciones informáticas como instrumento de conocimiento no tiene valor intrínseco. Es decir, su uso mecánico no garantiza la adecuación de conocimientos o hallazgos. Si su utilización se realiza dentro de un marco metodológico rutinario e irreflexivo que mistifica su aplicación, las simulaciones computerizadas no supondrían una innovación ventajosa ni más útil que cualquiera de las técnicas tradicionales en un marco de referencia tal.

Respecto a ello, lo que nos interesa destacar aquí es la utilidad de este tipo de desarrollos informáticos en su aplicación al análisis social, como mecanismos que favorecen la caracterización del dato complejo e instrumentos capaces de

3. Las relativas a la falta de disponibilidad de investigaciones con resultados no significativos en favor de la abundancia de análisis significativos. Existe, de hecho, un sesgo real en relación con la publicación de estudios con resultados significativos frente a los no significativos, lo que produce, a su vez, sesgos en el metaanálisis debido a la sobrerrepresentación de los primeros.

4. Las relativas a los efectos de interacción entre los resultados de los diferentes estudios. La falta de independencia de los resultados tiende a producir una aumento ficticio de la significación, ya que los resultados en la misma dirección aparecen sobrerrepresentados. 
aglutinar la cantidad de datos necesaria para abarcar la multidimensionalidad de la realidad social, concebida como sistema complejo. Sin caer en el apasionamiento y la fascinación que suelen generar este tipo de aproximaciones, ni tampoco en el enfervorecido rechazo que provoca en otros, consideramos las ventajas que suponen este tipo de simulaciones. Éstas permiten superar algunas de las limitaciones de la aplicación de los modelos matemático-estadísticos en la investigación social, en un doble sentido.

1. Por una parte, permiten recoger la multidimensionalidad social como ningún otro procedimiento $y$, del mismo modo, combinar regularidades con aspectos caóticos y de indeterminación ruido —elementos aleatorios y accidentales inherentes a cualquier proceso. Mayor o menor ruido hace referencia a la intensidad de estos elementos en el contexto en el que se desenvueleve la acción-. Las simulaciones hacen posible la configuración de lo que hemos dado en llamar el dato complejo.

2. Y, por otra parte, permiten superar la visión lineal de la vida social derivada de la asunción de la mayor parte de los análisis estadísticos tradicionales.

A pesar de ello y por su propia estructura, las simulaciones informáticas, aunque permiten, mejor que las técnicas tradicionales, tratar situaciones complejas, en la actualidad, sólo se vienen aplicando a niveles microsociológicos y de baja interacción ${ }^{11}$.

De este modo, sería ingenuo suponer que la utilización de simulaciones informáticas en el análisis de lo social es la solución definitiva a nuestros problemas metodológicos y técnicos. A pesar de que su aplicación supone una alternativa creativa y enriquecedora, ello no nos permite escapar del problema central de la debilidad teórico-conceptual de la sociología. De este modo y como ocurre con la aplicación general de los modelos matemático-estadísticos en la investigación social, en última instancia son los supuestos bajo los que se realiza su aplicación concreta los que condicionan de un modo u otro su desarrollo y sus resultados. Es decir, esta debilidad teórica condiciona y frena el desarrollo de una metodología que, a su vez, pueda fortalecer el corpus teórico. Inmersos al parecer en un círculo sin salida, la consideración de nuevas perspectivas, nuevos estilos metodológicos y elementos entre los que es posible establecer nuevas relaciones, se vislumbra como alternativa incitadora de nuevos procesos de teorización.

Con todo lo anterior, no hemos pretendido si no ofrecer diversos elementos que, aunque en cierto modo desestructurados, sirvan de base a una reflexión sólida del método en sociología. En definitiva, no intentamos más que una primera aproximación a un marco metodológico desde la complejidad. Conscientes de que la globalidad de esta idea puede producir ambigüedad y

11. Ver un ejemplo ilustrativo en Peter Kollock (1993) "An eye for an eye leaves everyone blind": cooperation and accouting systems», American Sociological Review, vol. 58, n. ${ }^{\circ} 6$. 
vaguedad respecto a algunos de los conceptos con ella asociados, y de la necesidad de profundizar en sus elementos estructurales, nuestro interés se centra en una concepción más flexible y abierta del investigar social. Su desarrollo sentido y fructificación estarán en función de los éxitos que puedan obtenerse a partir de esta nueva vía.

\section{Bibliografía}

AleXander, J.C. (1982). Theoretical Logic in Sociology. Vol. 1: Positivism, Presuppositions, and Current Controversies. Berkeley: University of California Press.

Anderson, Philip W. (1972). «More is different». Science, 177, p. 393-396.

ARAMBERRI, J.R. (1977). Los limites de la sociología burguesa. Madrid: Akal.

ARON, Raymond (1950). La philosophie critique de l'Historie. Essai sur une théorie allemande de l'Historie. París.

- (1970). Las etapas del pensamiento sociológico. Buenos Aires: Siglo XXI.

BACON, Francis (1961). Novum Organum. Estudio preliminar y notas de Risieri

Frondizi. Traducción directa del latín por Clemente Hernando Balmori, 2a ed.

Buenos Aires: Losada (ed. original en 1620).

Beltrán, Miguel (1979). Ciencia y sociología. Madrid: CIS.

Bemer, M. (ed.) (1981). Social Method and Social Life. Academic Press.

BERICAT, E. (1998). La integración de los métodos cuantitativo y cualitativo en la investigación social. Significado y medida. Arial Sociología. Madrid.

BlanK, G. (1989). «Introducción: New Technology and The Nature of Sociological Work». En New technology in sociology. Practical applications in research and work. Grant Blank.

Bоотн, Charles (1910). Poor Law Reform. Londres: Macmillan.

Bourdiel, P. y otros. El oficio del sociólogo. Madrid: Siglo XXI.

BRYMAN, A. (1984). «The Debate about Quantitative and Qualitative Research: A Question of Method or Epistemology?». The British Journal of Sociology, vol. XXXV, n. ${ }^{\circ}$ 1, p. 7-39.

Bryman, Alan; Duncan, Cramer (1990). Quantitative Data Analysis for Social Scientists. Londres y Nueva York: Routledge.

BRENT, E. (1984). "Qualitative Computing: Approaches and Issues». Qualitative Sociology, 7, p. 34-60.

BRIgGS, J.; PeAT, F.D. (1990). Espejo y reflejo: Del caos al orden. Guía ilustrada de la teoría del caos y la ciencia de la totalidad. Barcelona: Gedisa.

CAMPBElL, N.R. (1921). What is Science? Nueva York: Dover.

CAMPBELL, S. (1974). Equivocos y falacias en la interpretación de estadísticas. México: Limosa.

CARPAN; MORGERSTERm y otros. Aplicaciones de las matemáticas a las ciencias sociales y del comportamiento. Madrid: Alianza Editorial.

CARnaP, Rudolf (1966). Philosophical Foundations of Physics. Nueva York: Basic Books.

Cohen, P. (1980). «Efectiveness of student-rating feedback for improving college instructuion: A meta-analysis of findings». Research in Higer Education, n. ${ }^{\circ} 13$, p. 321-341.

Coleman, James (1964). Introduction to Mathematical Sociology. Nueva York: The Free Press.

- (1973). The mathematics of Collective Action. Chicago: Adine. 
Coluins, R. (1984). Statistics versus Words. Sociological Theory. San Francisco: Jossey-Bass. CONDE, Fernando (1987). «Una propuesta de uso conjunto de las técnicas cuantitativas y cualitativas en la investigación social. El isomorfismo de las dimensiones topoplógicas de ambas técnicas». Madrid: REIS, p. 213-224.

COOMBS, C.H.; RAIfFA, H.; Thrall, R.M. (1954). "Some views on Mathematical Model and Measurement Theory». Psychological Review, n. ${ }^{\circ}$ 61, p. 132-144.

- (1964). A Theory of data. Nueva York: John Wiley \& Sons.

- (1979). «Teoría y métodos de la medición social». En FestingER, L.; KATZ, D. Los métodos de investigación en las ciencias sociales. Buenos Aires: Paidós, p. 433-488.

COSER, Lewis. "Two Methods in Search of a Substance». American Sociological Review, n. ${ }^{\circ}$ 40, p. 691- 699 .

CHALMERS, Alan F. (1991). ¿Qué es esa cosa llamada ciencia? $1^{a}$ edición en español, enero de 1982. Madrid: Siglo XXI.

DAZINGER, J.M. (1989). «Waiting the revolution: the use of microcomputers by Social Scientists». En New technology in sociology. Practical applications in research and work. Grant Blank.

DenZIN, N. (1970). Sociological Methods. Chicago: Aldine.

DilTHEY, Wilhelm (1966). «Introducción a las ciencias del espíritu». Revista de Occidente, $2^{\text {a }}$ ed. Madrid.

Douglas, J. (1981). Investigative Social Research. Beverly Hills: Sage.

DurKHeIM, Emilio (1991). Las reglas del método sociológico. Madrid: Akal. Edición original 1895.

- (1990). Pragmatismo y sociología. Buenos Aires: Schafire SRL.

ECO, Umberto (1989). El signo de los tres. Barcelona: Lumen.

EMERSON, R. (1983). Contemporary Field Research. Boston: Little Brown.

EricKSON, F. (1986). "Qualitative Methods in Research on Teaching». En WitTrocK,

M.C. (ed.). Handbook of Research on Teaching. Nueva York: Mac Millan.

FEYERABEND, Paul (1981). Tratado contra el método. Madrid: Tecnos.

FOUCAULT, Michel (1972). La arqueología del saber. Madrid: Siglo XXI, 2a ed.

GaLtung, Johan (1966). Teoría y métodos de la investigación social. Buenos Aires: Eudeba.

- (1977). Methodology and Ideology. Essays in Methodology. Vol. 1. Copenague: Ejlers.

- (1979). Papers on Methodology. Vol. 2. Copenague: Ejlers.

- (1981). In defense of Epistemological Eclecticism. Ginebra: Institut Universitarie d'Études du Développment. Mimeo.

- (1995). Investigaciones teóricas. Sociedad y cultura contemporáneas. Madrid: Tecnos.

GARCÍA BLANCO, J.M. (1985). El problema de la irracionalidad en las ciencias sociales. Max Weber. Madrid: Tecnos.

GelL-Man, Murray (1999). El Quark y el jaguar. Aventuras de lo simple y lo complejo. Barcelona: Tusquets Editores.

GiBSON, Quentin (1968). La lógica de la investigación social. Madrid: Tecnos.

GLASS, G.V. (1976). «Primary, Secondary and Meta-Analysis of Research». Educational Researcher, n. ${ }^{\circ}$, p. 3-8.

Glass, G.; MCGaW, B.; Smith, M.L. (1981). Meta Analysis in Social Research. Beverly Hills, CA: Sage.

GÖDEL, Kurt (1980). «Sobre proposiciones formalmente indecibles de los Principia Mathematica y sistemas afines». Cuadernos Teorema, 8. Valencia: Departamento de Lógica, Universidad de Valencia.

GUZMÁN, Miguel y A. (1993). Estructuras fractales y sus aplicaciones. Barcelona: Labor. 
Haberman, S. (1979). Analysis of Quantitative Data. Nueva York: Academic Press. Hartwig, F. y Dearing, B.E. (1979). Exploratory Data Analysis. Beverly Hills, California: Sage. University Paper.

HEISENBERG, Werner (1958). Physics and philosophy: the revolution in modern science. Londres: Allen \& Unwin.

- (1949). The physical principles of the quantum theory. Nueva York: Dover.

HildeBrant, Stefan; TROMBA, Anthony (1990). Matemáticas y formas ópticas. Barcelona: Prensa Científica.

HorgAN, John (1995). «De la complejidad a la perplejidad». Investigación y Ciencia, n. ${ }^{\circ} 227$.

Hotelling, H. (1983). «Analysis of a Complex of Statistical Variables Intoprincipal Components». Journal of Educational Psychology, n. ${ }^{\circ}$ 24, p. 417-520.

HunTER, J.E.; SCHMIDT, F.L.; JACKSON, G.B. (1982). Meta-Analysis: Cumulating Research Findings Across Studies. Beverly Hills California: Sage.

IBÁÑ̄EZ, J. (1979). Más allá de la sociología. El grupo de discusión: Técnica y Crítica. Madrid: Siglo XXI.

- (1985a). "Análisis sociológico de textos y discursos». Revista. Internacional de Sociología, vol. 43, núm. 1, p. 119-160.

- (1985b). «Las medidas de la sociedad». REIS, n. ${ }^{\circ} 29$, enero-marzo. Madrid.

- (1986). Del algoritmo al sujeto. Perspectivas de la investigación social. Madrid: Siglo XXI.

IGLESIAS, M.C.; ARAMBERRI, J.R.; ZÚNIIGA, L.R. (1980). Los orígenes de la teoría sociológica. Madrid: Akal.

King, Gary; Keohane, Robert O.; Verba, Sidney (2000). El diseño de la investigación social. La inferencia científica en los estudios cualitativos. Madrid: Alianza Editorial. Ciencias Sociales.

KISH, L. (1954). «Some Statistical Problems in Research Design». American Sociological Review, n. 24 , p. 328-338.

- (1995). Diseño estadístico para la investigación. Madrid: CIS.

Kollock, P. (1993). "An eye for an eye leaves everyone blind": cooperation and accouting systems». American Sociological Review, vol. 58, n. ${ }^{\circ}$ 6, p. 768-786.

Kraemer, H.C.; ANDREWS, G. (1982). «A Nonparametric thecnique for meta- analysis effect size calculation». Psychological Bulletin, n. ${ }^{\circ}$ 91, p. 404-412.

KuHn, Thomas S. (1984). La estructura de las revoluciones cientificas. México: Fondo de Cultura Económica (1a edición: The Structure of Scientific Revolutions [1962]. University of Chicago Press).

LABOVITZ, S. (1970). «The Assingnment of Numbers to Rank Order Categories». American Sociological Review, n. ${ }^{\circ}$ 35, p. 515-524.

LAKATOS, I. (1974). Historia de la ciencia y sus reconstrucciones racionales. Madrid: Tecnos.

- (1986). La lógica del descubrimiento matemático. Madrid: Alianza Universidad. (Edición original: Proofs and Refutations - The Logic of mathematical Discovery [1976]. Cambrige University Press).

- (1987). Matemáticas. Ciencia y epistemología. Madrid: Alianza Universidad.

LAMO DE ESPINOSA, Emilio (1975). Juicios de valor y ciencia social. Valencia: Fernando Torres Editor.

- (1990). La sociedad reflexiva. Sujeto y objetivo del conocimiento sociológico. Madrid: Siglo XXI.

Landau, David; LaZARfeld, Paul (1977). "Quetelet, Adolphe». Enciclopedia Internacional de las Ciencias Sociales. Madrid: Aguilar, p. 55-61. 
LaZARSfeld, Paul F. (1959). Problems in Methodology. Nueva York: Sociology Today. Basic Books.

LAZARSFeld, Paul F.; Rosenberg, Morris (eds.) (1955). The Laguage of Social Research. Glencoe, ILL.: The Free Press.

Lebart, L.; Monrineau, A.; Fenelon, J.P. (1985). Tratamiento estadístico de datos. Métodos y programas. Barcelona: Marcombo

Le PlaY, Fréderic (1877-1879). Les ouvriers européens. 2a ed. 6 vols. Tours: Mame. Vol. I.: La methode d'observation appoliquée à l'etude des familles ouvrières. Edición original de 1855.

LEWIN, Roger (1995). El caos como generador de orden. Barcelona: Tusquets.

LIEBERSON, S. (1992). «Einstein, Renoir and Greeley: some thoughts about evidence en sociology». American Sociological Review, vol. 57, n. ${ }^{\circ}$ 1, febrero, p. 1-15.

LORENZ, Edward (1995). La esencia del caos. Un modelo cientifico para la disparidad de la Naturaleza. Barcelona: Debate.

Lyman, P. (1984). «Reading, Writing, and Word Processing». Qualitative Sociology, 7, p. 75-89.

MACY, Michael W. (1991). «Chains of Cooperation: Thershold Effects in Collective Action». American Sociological Review, vol. 56, n. ${ }^{\circ}$ 6, diciembre, p. 730-747.

- (1993). «Backward-Looking Social Control». American Sociological Review, vol. 58, n. ${ }^{\circ}$ 6, diciembre, p. 819-836.

MiLlS, W.C. (1957). The Sociological Imagination. Nueva York: Oxford University Press. (Versión castellana: La imaginación sociológica. 6a edición, 1974, México: Fondo de Cultura Económica).

MORIN, Edgar (1988). El método, el conocimiento del conocimiento. Cátedra. (Edición original: La Mêthode, III. La connaissance de la connaissance. Editors du Seuil, 1986).

- (1995). Introducción al pensamiento complejo. Barcelona: Gedisa.

Morrison, D.F. (1967a). Multivariate Techniques in Human Comunication Research.

- (1967b). Multivariate Statistical Methods. Nueva York: McGraw-Hill.

Nagel, E. (1967). «The Nature and Aim of Science». En Philosophy of Science Today. Nueva York: Basic Books.

- (1974). La estructura de la ciencia. Buenos Aires: Paidós.

OlTRA, Benjamín (1978). La imaginación ideológica. Una sociología de los intelectuales. Barcelona: Vicens-Vives.

- (1984). Naturaleza y sociedad para una ciencia abierta. Alicante: Universidad de Alicante

- (1995). Cultura y tiempo. Investigaciones de sociología de la cultura. Alicante: Aguaclara. Colección Amalgama.

ORTí, Alfonso (1994). "La confrontación de modelos y niveles epistemológicos en la génesis e historia de la investigación social». En DELGADO, J.M.; GUTIÉRREZ, J. (coords.). Métodos y técnicas cualitativas de investigación en Ciencias Sociales. Madrid: Síntesis Psicología, p. 85-95.

PETTY, William (1963). Political Arithmetick, vol. 1. The economic writings. N.Y. Kelley. Obra escrita alrededor de 1670 y publicada originalmente sin licencia. La $1^{\text {a }}$ edición, a título póstumo, fue autorizada por su hijo en 1690.

POPPER, Karl (1985). La lógica de la investigación cientifica. Madrid: Tecnos. Colección Estructura y Función. (1a edición: The Logic of Scientific Discovery [1962], Londres: Hotchinson \& Co. LTD.).

- (1985b). Realismo y objetivo de la ciencia. 
- (1985c). Teoría cuántica y el cisma en física. Madrid: Tecnos.

- (1986). El universo abierto. Un argumento en favor del indeterminismo. Madrid: Tecnos.

Prigogine, Ilya; NiCOLIS, Grégoire (1994). La estructura de lo complejo. Madrid: Alianza Editorial. Alianza Universidad.

QUeTELET, Adolphe (1869). Physique Sociale: Ou essair sur le développement des facultés: Physique Sociale. (Edición original, 1835). Chambers publicó en 1842 una traducción inglesa con el título: A Treatise on Man and the Development of this Faculties.

Rex, J. (1973). Discovering Sociology. Londres: Routledge and Kegan Paul.

- (1986). Problemas fundamentales de la teoría sociológica. Traducción de Néstor Mínguez. Rev. de Carlos Flood. Buenos Aires: Amorrurtu.

Rose, Gerry (1991). Deciphering Sociological Research. Theory, results, samples, concepts. Contemporany Social Theory. McMillan Education Ltd.

Rosenthal, R. (1980). «New Directions for Methodology of Social and Behavioral Science: Quantitative Assessment of Research Domain», n. ${ }^{\circ}$ 5. San Francisco: Jossey-Bass.

- (1984). Meta-Analysis Procedures for Social Research. Beverly Hills, California: Sage. Ruiz-Maya Perez, Luis y otros (1991). Metodología estadistica para el análisis de datos cualitativos. Madrid: CIS.

Ruiz Olabuenaga, J.I.; Ispizua, Marian (1989). La descodificación de la vida cotidiana. Bilbao: Universidad de Deusto.

RusSELL, Bertrand (1964). El conocimiento humano. Madrid: Taurus.

SCHRODT, Ph.A. (1984). Microcomputer methods for Social Scientists. Beverly Hills, California: Sage.

SchuTZ, A. (1964). Collected Papers II. La Haya: Nighoff.

SCHWARTZ, H.; JaCOBS, J. (1984). Sociología cualitativa. México: Trillas.

SIEGEL, S. (1983). Estadistica no paramétrica. México: Trillas.

SIERRA BraVO, R. (1983). Ciencias sociales. Epistemología lógica y metodología. Madrid:

Paraninfo.

Smith, M.W. (1975). Strategies of Social Research. New Jersey: Prentice Hall.

SNedeCor, G.W.; Cochran, W.G. (1967). Statistical methods. 6a edición. Ames, Iowa: Iowa State University Press.

STEVENS, S.S. (1946). "On the Theory of Scales of Measurement». Science, n. ${ }^{\circ}$ 103, p. 677-680.

STRAUSS, A. (1987). Qualitative Analysis. Cambridge.

TREND, M.G. (1987). «On the Reconciliation of Qualitative and Quantitative Analisys: a case study». Human Organization, vol. 37, núm. 4, p. 345-365.

TUKEY, J.W. (1949). «One degree of freedom for nonadditivity». Biometrics, n.o 5 , p. 232-242.

- (1977). Exploratory Data Analysis. USA. Addison-Wesley Publishing Company. Van MaAnen, J. (1983). Qualitative Methodology. Londres: Sage.

WAGENSBERG, Jorge (1993). Ideas sobre la complejidad del mundo. Barcelona: Tusquets. $1 .^{\mathrm{a}}$ ed., 1985.

WallaCe, Walter L. (1976). La lógica de la ciencia en sociología. Madrid: Alianza Universidad.

- (1983). Principles of Scientific Sociology. Aldine Publishing Company.

WARTOFSKY, Mark W. (1983). Introducción a la filosofía de la ciencia. Madrid: Alianza Universidad Textos.

WeBER, M. (1922). Gesammelte Aufsätze zur Wissenschaftslehre. J.C.B. Morh, Tubinga. 
- (1958). Ensayos sobre metodología sociológica. Buenos Aires: Amorrortu.

- (1972). Ensayos de metodología contemporánea. Barcelona: Martinez Roca.

- (1974). Sobre la teoría de las ciencias sociales. Barcelona: Península.

WINCH, R.F.; MoORE, D.M. (1956). «Quantitative analisys of Qualitative data in the assesment of motivation: reliability congruence and validity». American Journal of Sociology.

WOLF, Frederic M. (1986). Meta Analysis in quantitative methods for research synthesis. California U.S.A: Sage Publications Inc. Series quantitative aplications in the social sciences. A Sage University Paper.

WoOlley, Benjamin (1992). Virtual worlds. Oxford, Cambridge: Blackwell.

WrigTH, G.H. Von (1958). Explanation and Understanding. Routledge and Kegan Paul. Edición en castellano: Explicación y comprensión. Madrid: Alianza. 2a edición, 1987.

Wrigth Mills, C. (1993). La imaginación sociológica. Madrid: Fondo de Cultura Económica.

ZEISEL, Hans (1962). Diálogo con números. Buenos Aires: Fondo de Cultura Económica. ZnANIECKI, F. (1934). The Method of Sociology. Nueva York: Farrer. 